Tropical Journal of Pharmaceutical Research October 2016; 15 (10): 2173-2182

ISSN: $1596-5996$ (print); 1596-9827 (electronic)

(C) Pharmacotherapy Group, Faculty of Pharmacy, University of Benin, Benin City, 300001 Nigeria.

All rights reserved.

Available online at http://www.tjpr.org

Original Research Article

http://dx.doi.org/10.4314/tjpr.v15i10.16

\title{
Neuroprotective effect of paeonol against isoflurane- induced neuroapoptosis and cognitive dysfunction
}

\author{
Jian-Xin Zhang*, Zhi-Ying Li, Liang Zhao, Gang Li, Gui-Lin Cao, Chuan-Yang \\ Zhang \\ Department of Anesthesiology, The 148th Central Hospital of Chinese People's Liberation Army, Zhoucun District, Zibo, \\ Shandong 255300, P.R. China
}

*For correspondence: Email: jianxinjx@hotmail.com; Tel/Fax: 0086-0533-6552118

Revised accepted: 8 September 2016

\begin{abstract}
Purpose: To investigate whether paeonol affords neuroprotection against isoflurane-induced neurotoxicity.

Methods: Separate groups of neonatal rat pups were administered paeonol $(20,40$ or $80 \mathrm{mg} / \mathrm{kg})$ from post-natal day 3 (P3) to post-natal day 15. On post-natal day 7, the pups were exposed to $6 \mathrm{~h}$ of isoflurane $(0.75 \%)$ anesthesia. TUNEL assay was performed to assess neuroapoptosis. Cleaved caspase-3 expressions were evaluated by immunohistochemistry and western blotting analysis. The expressions of apoptotic pathway proteins and mitogen activated protein kinases (MAPKs) were assessed by western blotting. The general behaviour of the rats was determined by open field test and elevated maze test. Y-maze test and Morris water maze tests were performed to evaluate working memory and cognition.

Results: Isoflurane exposure caused $(p<0.05)$ severe neuronal apoptosis in the hippocampal region and enhanced caspase-3 expressions. Paeonol supplementation remarkably $(p<0.05)$ reduced neuronal apoptosis and modulated expressions of apoptotic proteins. The raised expressions of NF-KB, TNF- $\alpha$, IL-6 and IL-1 $\beta$ and significantly $(p<0.05)$ enhanced JNK/p38 signalling cascades were inhibited by paeonol. The expression levels of ERK were not significantly $(p<0.05)$ changed, but there was significant improvement in the general behaviour and working memory of the rats.

Conclusion: Paeonol significantly improves cognitive impairments and offers neuroprotection against isoflurane-induced apoptosis via modulating JNKJERK/p38 MAPK and NF-KB signaling pathways.
\end{abstract}

Keywords: Apoptosis, Isoflurane, Neurodegeneration, Paeonol, Cognitive impairment, Signaling pathways

Tropical Journal of Pharmaceutical Research is indexed by Science Citation Index (SciSearch), Scopus, International Pharmaceutical Abstract, Chemical Abstracts, Embase, Index Copernicus, EBSCO, African Index Medicus, JournalSeek, Journal Citation Reports/Science Edition, Directory of Open Access Journals (DOAJ), African Journal Online, Bioline International, Open-J-Gate and Pharmacy Abstracts

\section{INTRODUCTION}

Increasing number of adults and children are exposed to anaesthetics as a part of medical care including surgery or related procedures [1]. Volatile anesthetics sevoflurane and isoflurane are commonly used general anesthetics. However, extended exposure to anesthesia can cause widespread neurodegeneration in the developing brains. Anaethesia-induced with long- term learning deficits and memory impairments have also been reported [2,3]. Recent retrospective studies have revealed that children exposed to anesthetics during early years are at higher risks of cognitive and behavioural disabilities [4].

Previous studies have shown that isoflurane causes severe neuroapoptosis in both 
developing animal brains and primary neuronal cells [3]. Neuroinflammation has been reported to be involved in volatile anesthetic-induced neuroapoptosis and cognitive impairments [5]. Isoflurane induces striking increase in intracellular calcium levels $\left[\mathrm{Ca}^{2+}\right] \mathrm{i}$ and this overload has been found to activate mitochondrial apoptotic pathway [6] and also the c-Jun N-terminal kinase (JNK) [2].

The ERK, p38 MAPK and JNK are major members of mitogen-activated protein kinases (MAPK). MAPK cascades critically regulate neurogenesis [7], neurodegeneration [8], pain and brain inflammation [9] under normal and pathological conditions. SP600125, a selective JNK inhibitor] was reported to exert neuroprotective effects in neurodegenerative diseases [10]. Furthermore, it is well known that p38 MAPK plays an important role in regulation of apoptosis and cell cycle arrest [11]. Thus, targeting JNK/ERK/p38 MAPK signalling cascades is of immense benefit in preventing isoflurane-mediated neuroapoptosis.

Paeonol is a major phenolic compound of the genus Paeonia and a key active principle of plants such as Paeonia albiflora and Paeonia moutan. Paeonol possesses wide range of biological properties such as anti-inflammatory [12], antitumor [13] and neuroprotective effects [14]. Paeonol has been found to prevent cardiovascular diseases [15]. . Considering the pharmacological potential of paeonol, we attempted to investigate if paeonol offered protection against isoflurane-induced neuroapoptosis and cognitive dysfunctions in neonatal rats.

\section{EXPERIMENTAL}

\section{Chemicals and reagents}

Isoflurane $(0.75 \%)$ and paeonol were obtained from Sigma-Aldrich (St. Louis, MO, USA). Antibodies against cleaved caspase-3, Bcl-2, Bad, Bcl-xL, Bax, inhibitors of apoptosis proteins - $x I A P$ and clAP-1, survivin and $\beta$-actin were obtained from Cell Signalling Technology (Beverly, MA, USA). JNK, ERK, p38, phosphoJNK, phospho-ERK, phosphor-p38 and phosphoc-Jun, NF-kBp65, p-IкBa, TNF- $\alpha$, IL-1 $\beta$ and IL-6 (Santa Cruz Biotechnology, Santa Cruz, CA, USA) were used for expression analysis. All other chemicals and reagents used in this study are of analytical grade and were purchased from Sigma-Aldrich (St.Louis, MO, USA) otherwise are mentioned.

\section{Animals}

Pregnant Sprague-Dawley rats (Guangdong Medical Laboratory Animal Co., China) were housed in sterile plastic cages under standard housing conditions of $12 \mathrm{~h}$ day/night cycle and were maintained at $23 \pm 2{ }^{\circ} \mathrm{C}$. The rats were supplied with water and standard pellet ad libidum. The animals were monitored closely for the delivery of pups. The day of birth of rat pups was noted as post-natal day 0 (P0). The rat pups were maintained under the same conditions indicated above. The study was approved by the University Animal Care Committee (approval no. 2013034543) and performed in compliance with National Institutes of Health Guide for the Use of Laboratory Animals [16].

\section{Isoflurane dosing}

Separate groups of rat pups were administered paeonol at 20,40 or $80 \mathrm{mg} / \mathrm{kg}$ body weight from P3 to P15 along with standard diet. On P7, pups were exposed to $0.75 \%$ isoflurane for $6 \mathrm{~h}$ (approximately $0.3 \mathrm{MAC}$ in $\mathrm{P} 7$ rat pups as described by Orliaguet et al [17]) in $30 \%$ oxygen or air in a temperature controlled chamber. P7 rats were chosen based on previous studies suggesting that the period is most vulnerable to anesthesia-induced neuronal damages. Control rats received no anesthesia or paeonol.

\section{Assessment of neuroapoptosis - TUNEL fluorescent assay}

The influence of paeonol at different concentrations $(20,40$ or $80 \mathrm{mg})$ on neuroapoptosis induced by isoflurane was assessed by TUNEL assay. The rat pups, following isoflurane exposure, were perfused transcardially with ice-cold saline followed by $4 \%$ paraformaldehyde in $0.1 \mathrm{M}$ phosphate buffer. The brain tissues were then post-fixed for $48 \mathrm{~h}$ at $4{ }^{\circ} \mathrm{C}$ and are embedded in paraffin. The tissues were sectioned at $5 \mu \mathrm{m}$ thickness. The TUNEL assay was performed using Dead End TM fluorometric TUNEL system kit (Promega, Madision, WI, USA). TUNEL positive cells in the hippocampal CA1, CA3 and dentate gyrus (DG) regions were analyzed with NIS-Elements BR image processing and analysis software (Nikon Corporation, Japan).

\section{Immunohistochemistry}

To further assess isoflurane-induced neuroapoptosis cleaved caspase-3 expression was analysed in brain tissues by immunohistochemistry as described previously by Li et al [18]. Furthermore, the brain sections were incubated 
with anti-cleaved caspase-3 primary antibody at $4{ }^{\circ} \mathrm{C}$ overnight following incubation with secondary antibody (Santa Cruz Biotechnology, Santa Cruz, CA, USA) for 40 min. After treatment with avidin-biotinylated peroxidase complex (Vectostain ABC-Kit, Vector Lab, Burlingame, CA, USA) for $40 \mathrm{~min}$, brain tissue sections were treated with diaminobenzidine and analyzed with NIS-Elements BR image processing and analysis software. Extend of apoptosis was determined as caspase positive cell counts in the CA1, CA3 and DG regions.

\section{Immunoblotting}

The expression of apoptotic pathway proteins and MAPK proteins in the hippocampi of the rat pups were analysed by western blotting. The analysis was carried as previously described by Li et al [18]. Brain tissues were homogenized in ice-cold RIPA buffer (50 mM Tris, pH 7.2; $1 \%$ sodium deoxycholate; $150 \mathrm{mM} \mathrm{NaCl} ; 0.1 \%$ sodium dodecyl sulfate (SDS); $10 \mathrm{mM} \mathrm{NaF;} 1 \%$ Triton X-100; $1 \mathrm{mM} \mathrm{Na3VO4;} \mathrm{protease} \mathrm{inhibitor}$ cocktail (1:1000)]. Homogenates were centrifuged at $14,000 \mathrm{rpm}$ for $10 \mathrm{~min}$ at $4{ }^{\circ} \mathrm{C}$ and the supernatants were collected. Protein concentration in the tissue samples were determined by BCA protein assay (Bio-Rad, Hercules, CA, USA). Equal amounts $(60 \mu \mathrm{g})$ of protein samples was subjected to electrophoresis on SDS-PAGE and blotted on to polyvinylidene difluoride membranes. The membranes were incubated with respective primary antibodies at 4 ${ }^{\circ} \mathrm{C}$ overnight, washed and were further incubated with appropriate secondary antibodies. The immunoreactive bands were visualized and scanned using Image Master II scanner (GE Healthcare, Milwaukee, WI, USA). The densities of the bands were further analyzed by ImageQuant TL software v2003.03 (GE Healthcare, Milwaukee, WI, USA). The protein expressions were normalized to $\beta$-actin.

\section{Memory and learning studies}

The effects of paeonol treatment on isofluraneinduced behavioural changes were assessed by the following tests.

\section{Open-field test}

Open-field test was performed to assess the behavioural response of the rats to a novel environment. P32 rats exposed to isoflurane on P7 were subjected to open-field test as previously described [19]. The behavior of the rats in the novel environment was noted as the movement of the rats in the open field. The total distance travelled (meters) in the test span of 10 min was recorded. The movement of the rats were monitored and analyzed using a computerized video tracking system (SMART, Barcelona, Spain).

\section{Elevated plus-maze test}

In order to evaluate anxiety-related behaviour, the elevated plus-maze test was conducted as described by Satoh et al [19]. The apparatus used for the test consisted of two open ( $25 \times 5$ $\mathrm{cm}$ ) and two enclosed arms. The arms were placed at $50 \mathrm{~cm}$ above the floor. The percentage of time spent in the open arm by the rats for a period of $10 \mathrm{~min}$ was noted and this was considered as anxiety index. The responses of the rats were recorded using the computerized video tracking system (SMART, Barcelona, Spain).

\section{Y-maze test}

The Y-maze test was performed to evaluate the spatial working memory. The apparatus consisted of a symmetrical Y-maze with three arms $(25 \times 5 \mathrm{~cm})$ separated by 15-cm-high transparent walls. The P32 rats exposed to isoflurane on P7 were placed at the centre of the maze and were allowed to freely explore the maze. Each rat was given a time of $8 \mathrm{~min}$ to explore the maze and the total number of times the rats entered the arms and sequence of entry were noted. The memory was assessed as reported earlier by Kodama et al [20].

\section{Morris water maze test}

The influence of paeonol on isoflurane-induced alterations in memory and learning were evaluated using Morris water maze (MWM). The experiments were performed as described previously by Li et al [18].

P7 rat pups exposed to isoflurane and/or paeonol were trained for a period of 4 days between P31 and P35 in the Morris water maze. A platform of about $10 \mathrm{~cm}$ in diameter was submerged in a circular pool (200 cm diameter, $60 \mathrm{~cm}$ depth) filled with warm water $\left(23 \pm 2{ }^{\circ} \mathrm{C}\right)$. The rats were subjected to 2 training sessions/day. The rats were allowed to take $60 \mathrm{~s}$ to locate the hidden platform in the pool and if they failed to locate in the given time, they were gently guided. Performance of the rats and swim paths were monitored and recorded using ANY-maze video tracking system (Stoelting Co., Wood Dale, IL, USA). The tracking system records and measures the time taken (latency) by each rat to find the platform. 


\section{Cued trials}

P35 rats were subjected to cued trials to assess any visual impairments and/or swimming difficulties. The pool was covered by a white cloth to hide visual cues. The rats were given 4 trials/day. During each trial, the rats were placed in a specific position of the pool and allowed to swim to the platform that had a rod attached. The rod served as cue and was placed approximately $20 \mathrm{~cm}$ above water level in any one of the quadrants of the pool. Rats were allotted $60 \mathrm{sec}$ to locate the submerged platform with the help of the rod and about $30 \mathrm{sec}$ time to sit on the platform. The rats that were unable to locate the platform within $60 \mathrm{sec}$ were gently guided and allowed to remain there for $30 \mathrm{sec}$. The time taken by rats to reach the cued platform was recorded.

\section{Place trials}

The white cloth used for cued trials was removed and same set of rats were assessed for their ability to learn and remember the spatial relationship between cues and the submerged platform (with no cue rod). The platform was placed in the same position throughout the place trials. The rats were placed at random starting points and the time taken to reach the platform without the cue rod was noted.

\section{Probe trials}

Probe trials were conducted $24 \mathrm{~h}$ after place trials to assess memory. The submerged platform was removed from the target quadrant (the quadrant in which the platform was kept throughout the place trials). Rats were allowed to swim for $60 \mathrm{sec}$ in the pool and the time that each rat spent looking out for the platform in the target quadrant was recorded. The time spent by the rats in the target quadrant in comparison to the time spent in other quadrants was considered as indication of memory retention.

\section{Statistical analysis}

The results are presented as mean \pm standard deviation (SD), taken from three or six independent experiments and were subjected to one-way analysis of variance (ANOVA) and Duncan's Multiple Range Test (DMRT) using SPSS statistical package (version 22.0). $P<0.05$ was considered statistically significant.

\section{RESULTS}

\section{Paeonol reduces isoflurane-induced neuroapoptosis}

In present study, isoflurane exposure significantly $(p<0.05)$ raised apoptosis in $\mathrm{P} 7$ rats. Paeonol administration brought marked decrease $(p<$ 0.05 ) in apoptotic cell counts in the hippocampalCA1, CA3 and in dendate gyrus (DG) regions. The decrease was dose-dependent with $80 \mathrm{mg}$ dose exhibiting maximum effects (Figure 1).

Isoflurane increased $(p<0.05)$ the expression of cleaved caspase-3 and it was noticed that paeonol markedly $(p<0.05)$ reduced caspase-3 expression (Figure 2). Quantification of cleaved caspase-3 protein expression by western blots also revealed significantly enhanced $(p<0.05)$ expression following isoflurane exposure (Figure $3)$. Paeonol at the tested doses (20, 40 and 80 mg) significantly down-regulated caspase-3

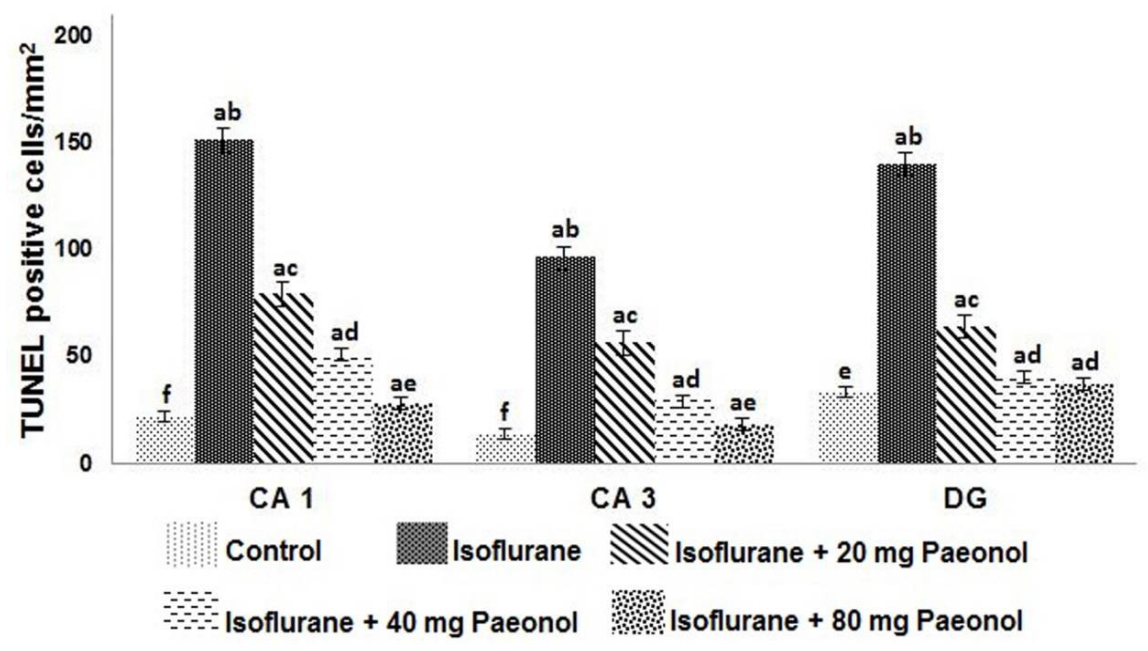

Figure 1: Paeonol inhibits neuroapoptosis induced by isoflurane. Values are presented as mean $\pm S D, n=6 .{ }^{a}$ represents statistical significance at $p<0.05$ compared against control and ${ }^{\text {b-f }}$ represents mean values within the same group that differ from each other at $p<0.05$ as determined by one-way ANOVA followed by DMRT analysis 
protein expression. This suppression in part could have contributed to the decrease in apoptotic cell counts.

\section{Paeonol modulates expressions of apoptotic pathway proteins}

Six hours of isoflurane exposure in $\mathrm{P} 7$ rat pups caused a robust $(p<0.05)$ increase in the expression of Bax and Bad with decreased expression of $\mathrm{Bcl}-2$ and $\mathrm{Bcl}-\mathrm{xL}$ (Figure 3). Administration of paeonol presented significant down-regulation of Bax and Bad, while upregulating the levels of $\mathrm{Bcl}-\mathrm{xL}$ and $\mathrm{Bcl}-2$. The higher doses of paeonol (40 and $80 \mathrm{mg}$ were more effective than $20 \mathrm{mg}$ dose.

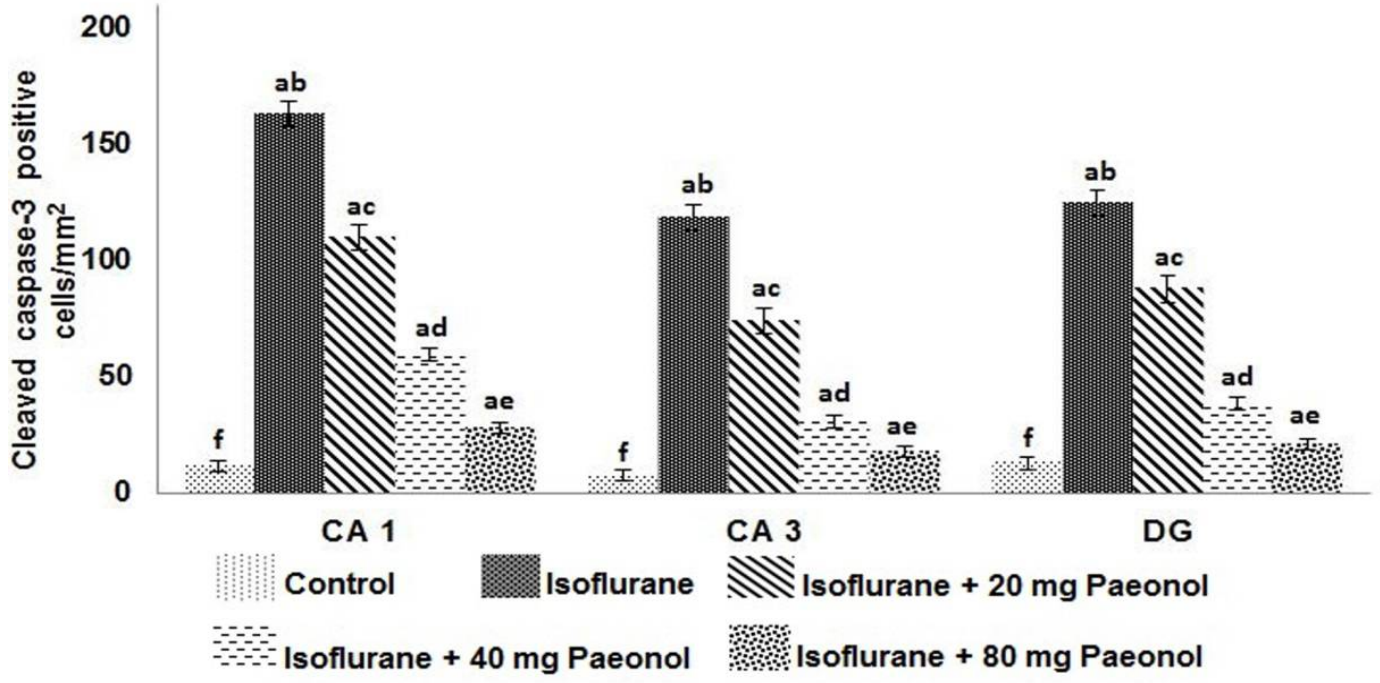

Figure 2: Influence of paeonol on caspase -3 expressions. Paeonol significantly reduced Isoflurane enhanced caspase-3 expressions in a dose-dependent way. Values are represented as mean $\pm S D, n=6 .{ }^{a}$ represents statistical significance at $p<0.05$ compared against control and ${ }^{\text {b-f }}$ represents mean values within the same group that differ from each other at $p<0.05$ as determined by one-way ANOVA followed by DMRT analysis

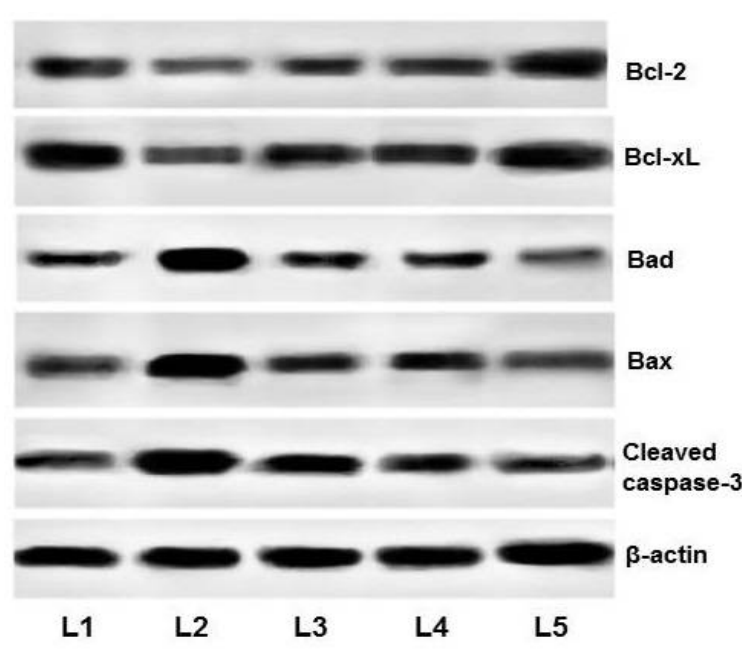

Figure 3: Paeonol modulates the expression of apoptosis pathway proteins. Isoflurane-induced enhanced expressions of proapoptotic proteins were down-regulated by paeonol (L1-control; L2-isoflurane; L3-isoflurane + $20 \mathrm{mg}$ paeonol; L4-isoflurane $+40 \mathrm{mg}$ paeonol; L5-isoflurane $+80 \mathrm{mg}$ paeonol)

Influence of paeonol on the expression of proteins of the MAPK signalling pathways

Isoflurane significantly $(p<0.05)$ enhanced the expression of JNK and p38 and as well the phosphorylated forms of JNK and p38 MAPK (Figure 4). Nevertheless not much increased expression of ERK and p-ERK were found following isoflurane exposure. On paeonol administration, down-regulation of JNK and p38 was observed along with reduced $\mathrm{p}-\mathrm{JNK}$ and $\mathrm{p}$ 38 expressions. Pre-treatment with paeonol at 20, 40 or $80 \mathrm{mg}$ doses failed to cause much changes in the expression levels of ERK and pERK compared to the suppression of JNK and p38 MAPK. While 20 and $40 \mathrm{mg}$ doses did cause down-regulation of $p$-JNK and p-p38, significant results were observed with $80 \mathrm{mg}$ paeonol.

\section{Paeonol significantly regulates the NF-KB signalling}

Enhanced expression of TNF- $\alpha$, NF-kBp65, pIKB $\alpha, I L-1 \beta$ and IL- 6 on isoflurane exposure with lowered expression of xIAP, clAP and survivin were observed (Figure 5). Paeonol was found to enhance expression of xIAP and cIAP. Paeonol significantly down-regulated the NF-KB signalling cascades, by effective inhibition of TNF- $\alpha, I L-1 \beta$ and IL-6.

Trop J Pharm Res, October 2016; 15(10): 2177 


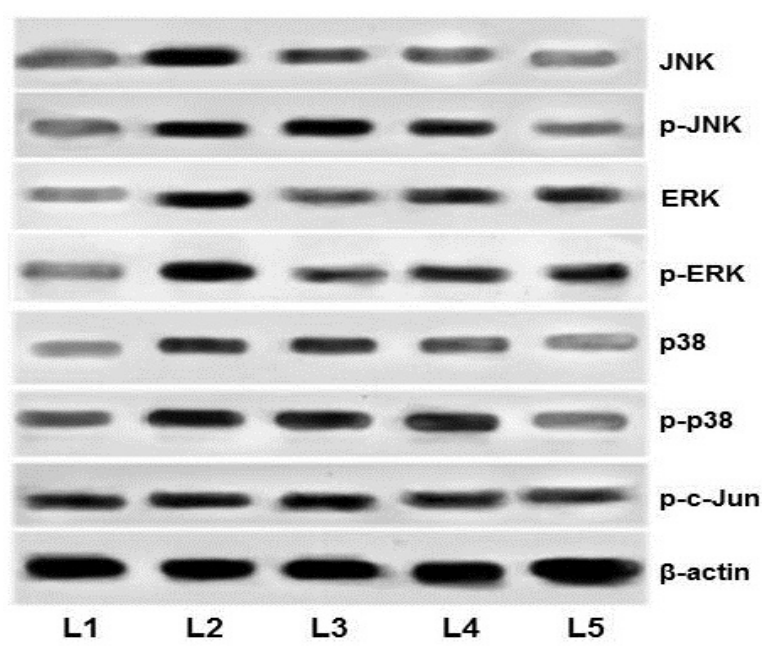

Figure 4: Paeonol modulates the expression of MAPK pathway proteins. Isoflurane enhanced JNK/p-38/ERK expressions were significantly down-regulated by paeonol (L1-Control; L2-Isoflurane; L3-Isoflurane + 20 mg paeonol; L4-Isoflurane + $40 \mathrm{mg}$ paeonol; L5Isoflurane $+80 \mathrm{mg}$ paeonol)

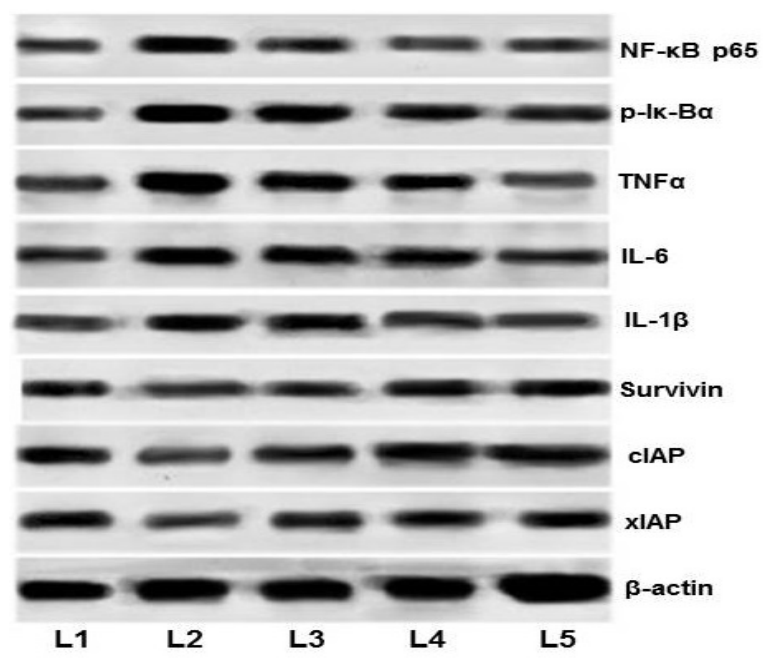

Figure 5: Paeonol modulates NF-kB signalling pathway. Paeonol down-regulated isoflurane-induced activation of NF-KB mediated inflammatory responses (L1-control; L2-isoflurane; L3-isoflurane $+20 \mathrm{mg}$ paeonol; L4-isoflurane $+40 \mathrm{mg}$ paeonol; L5-isoflurane $+80 \mathrm{mg}$ paeonol)

\section{Influence of paeonol on behaviour and memory of rats following exposure to isoflurane}

In the open field test, the behaviour of rats in a novel environment was observed. Isoflurane alone-exposed rats exhibited no noticeable behavioural disturbances, however the distance they moved across the new open field was considerably less than the rats that were treated with paeonol and exposed to isoflurane. The rats that received paeonol at 40 and $80 \mathrm{mg}$ doses exhibited behaviour almost similar to control rats that received no paeonol or isoflurane (Figure $6 a)$.

The elevated maze test revealed that rats administered only isoflurane spent considerably $(p<0.05)$ lesser time in the open arms when compared to control. Paeonol treatment significantly $(p<0.05)$ improved the behaviour of the animals. Paeonol treated rats spent more percentage of time in the open arms in the given 10 min of test time, suggesting improvements in the behaviour of animals (Figure 6b).

In order to examine whether isoflurane exposure affected the spatial working memory, we performed the Y-maze test. The choice and the sequence of arms selected by the rats were recorded. We observed indifferent behaviour in terms of the arms attempted by the rats exposed to isoflurane as compared to control rats. Paeonol at all the three tested doses was found to considerably improve the performance of the rats (Figure 6c).

\section{Learning and memory of animals}

The time taken by each rat to navigate the pool and reach the platform was recorded as escape latency. In the present study we found that the latency time decreased for all animals with every training session irrespective of paeonol administration (Figure 7a). However the rats administered isoflurane alone exhibited smaller variation when compared to animals that received paeonol and exposed to isoflurane.

Rats exposed to isoflurane alone were observed to take considerably a much longer time to reach the submerged platform with the rod (cue) when compared to control rats that received no anesthesia or paeonol. Nevertheless, the animals that received paeonol took significantly lesser duration to reach the platform as against rats that received isoflurane alone. Also, rats that received higher dose of paeonol $(80 \mathrm{mg})$ exhibited maximum improvement in performance (Figure $7 b$ ). Further, place and probe trials were conducted to assess the ability of the rats to locate and as well remember the location of the submerged platform without cue rod. In place trials, paeonol-supplemented-rats presented improved performance by taking significantly lesser time to reach the platform.

Further memory retention was evaluated by placing the submerged platform in a random 


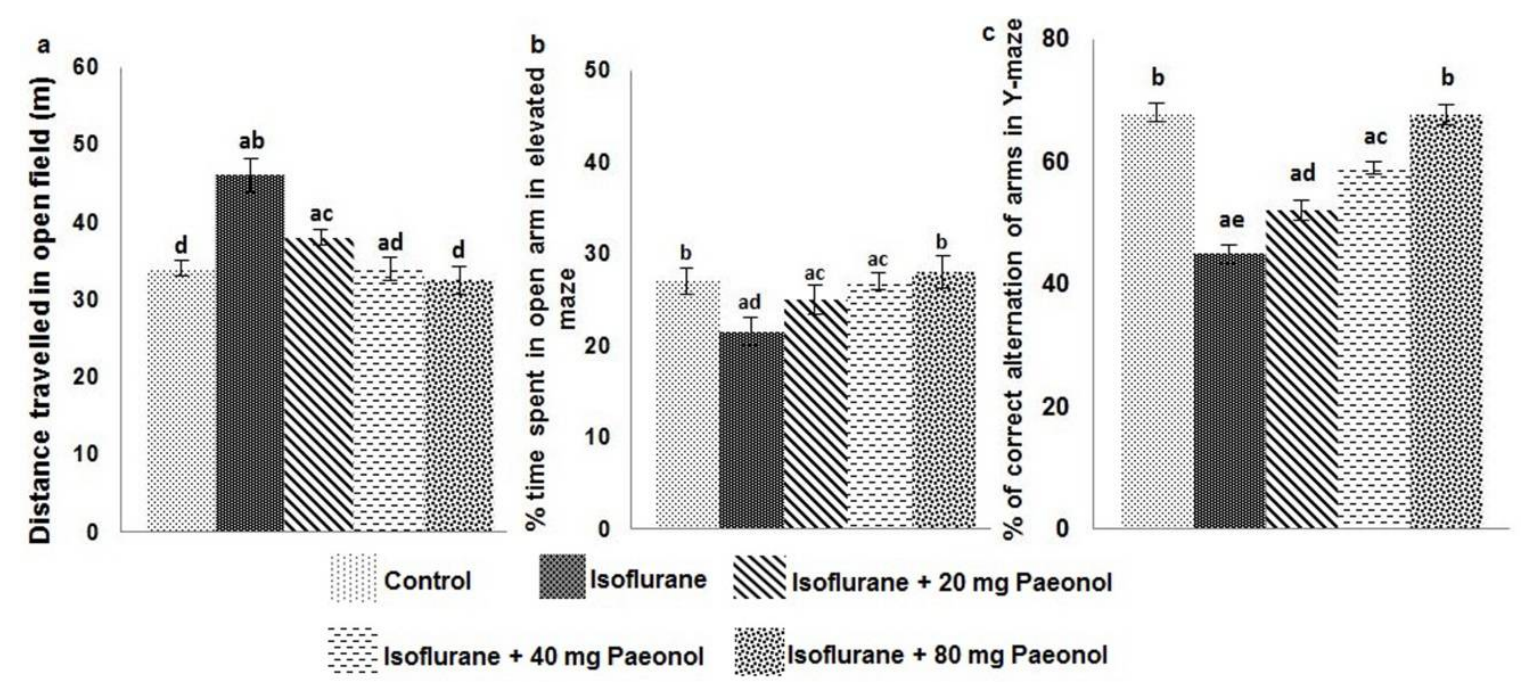

Figure 6: Effect of paeonol on behaviour of rats following exposure to isoflurane on P7. Isoflurane-induced memory impairments and cognitive deficits were significantly improved by paeonol. Paeonol at all the tested doses markedly enhanced the performance of rats subjected to novel environment (a) elevated maze (b) Y-maze test (c) .Values are represented as mean $\pm \mathrm{SD}, \mathrm{n}=6$. ${ }^{\mathrm{a}}$ represents statistical significance at $p<0.05$ compared against control and ${ }^{\text {b-f }}$ represents mean values within the same group that differ from each other at $p<0.05$ as determined by one-way ANOVA followed by DMRT analysis

(b)

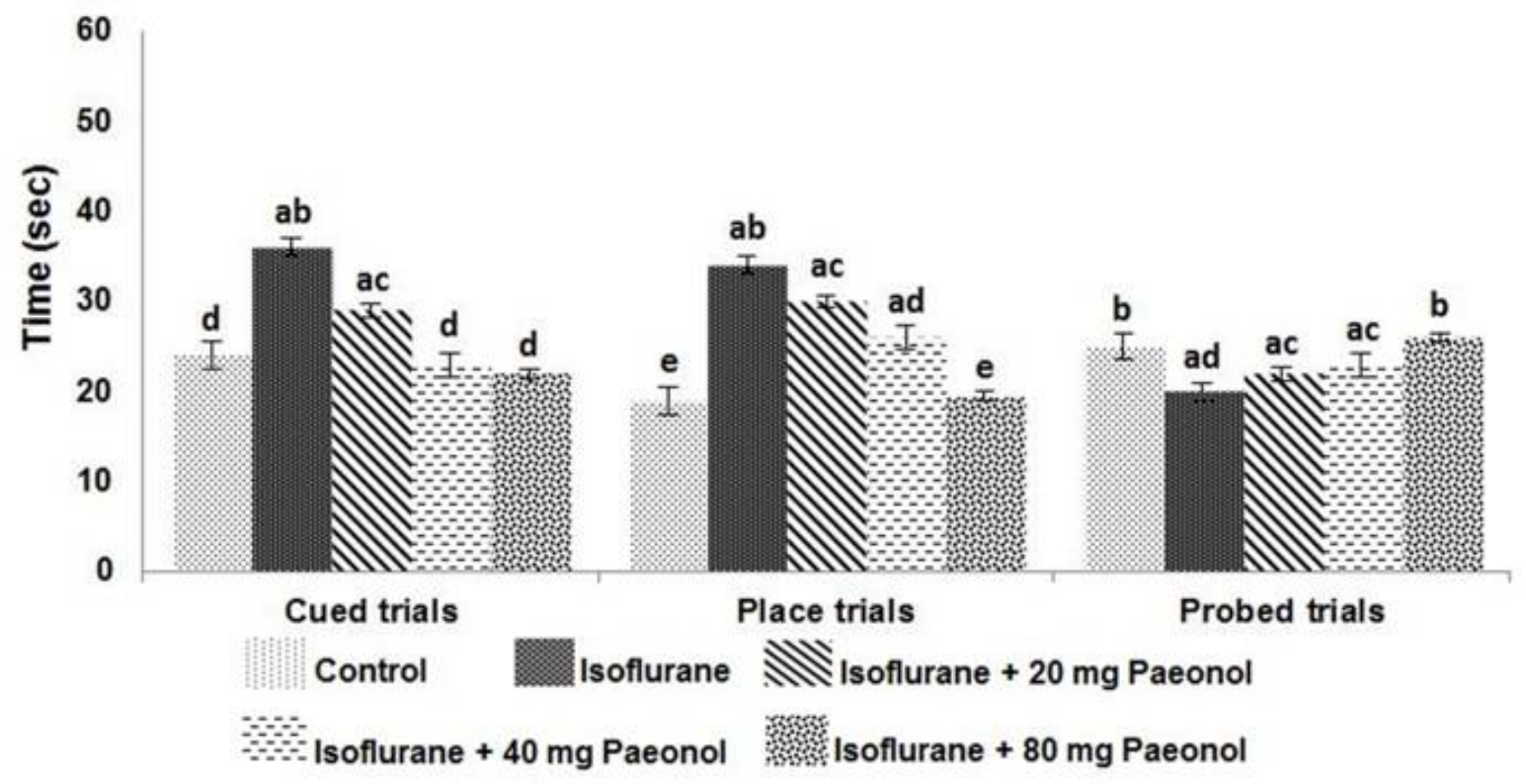

Figure 7: Paeonol improved learning and memory of rats exposed to isoflurane on P7. (a) Escape latency of the rats exposed to isoflurane. (b) Paeonol effectively improved learning and memory of P35 rats following anesthesia exposure on P7 as determined by cued, place and probe trials with Morris Water maze. Values are represented as mean \pm SD $(n=6)$; ${ }^{a}$ represents statistical significance at $p<0.05$ compared against control and b-f represents mean values within the same group that differ from each other at $p<0.05$ as determined by oneway ANOVA followed by DMRT analysis

quadrant other than the target quadrant. Duration of time taken to look out for the platform at the target quadrant was recorded.

Anesthesia exposure was observed to have a significant impact on memory of the rats, as the rats exposed to isoflurane spent considerably ( $p$
$<0.05)$ lesser time in the target quadrant as compared with control group rats that were not exposed to anesthesia (Figure 7b). Memory retention was found to be improved in paeonoltreated-rats. The P35 rats administered paeonol were observed to spend more time in the target quadrant looking out for the platform. Higher 
doses of paeonol were found to be more effective in improving the performance of the rats than the lower dose of $20 \mathrm{mg}$. The observations suggest that paeonol was able to significantly improve memory and cognition of the rats.

\section{DISCUSSION}

Isoflurane either alone or combined with other drugs is frequently used as general anesthesia in children, and adults. Increasing experimental data demonstrate that isoflurane induced neuronal apoptosis in the developing brains and also caused long-term neurocognitive dysfunctions [21]. Thus compounds that could effectively counter the toxic effects of isoflurane without disturbing its anesthetic potential could be of great value in medicine.

In line with the previous studies we observed robust increase in apoptotic cell counts in the hippocampal regions of $\mathrm{P} 7$ rats exposed to $6 \mathrm{~h}$ of isoflurane. Though anesthetic-induced neuroapoptosis occurs widely in the brain, we focused on the hippocampal region, since studies have demonstrated that hippocampal neurogenesis is associated with memory acquisition [1]. In our study we observed remarkable reduction in apoptosis counts in CA1, CA3 and DG regions of the pups that were supplemented with paeonol. Further immunohistochemistry and expression analysis revealed significantly enhanced caspase-3 expressions following exposure to isoflurane. Previous studies have demonstrated increased activated caspase-3 expressions on isoflurane exposure indicating cellular apoptosis [3]. Cleaved caspase-3 expression is considered as a marker of apoptosis and cell death in anesthetic injury [22]. This activation may contribute to the structural changes in brain tissues [3].

Enhanced expression of pro-apoptotic proteins Bad and Bax observed after isoflurane treatment suggest neuroapoptosis. The balance between pro-apoptotic factors and anti-apoptotic factors is very critical in cell survival. Paeonol administration was found to profoundly bring back the balance by effectively improving the expressions of $\mathrm{Bcl}-2$ and $\mathrm{Bcl}-\mathrm{xL}$ while downregulating Bad and Bax. Studies have reported that $\mathrm{Bcl}-\mathrm{xL}$ maintains mitochondrial membrane integrity and also inhibits cytochrome c release thus improving cell survival [23] and suppression of apoptosis. In this study activated caspase-3 expression was also reduced by paeonol. These effects potentially could have contributed to the decreased apoptotic cell counts observed.
Isoflurane has been reported to induce neuroinflammation [5]. More notably, IL-1 $\beta$ is associated with isoflurane-induced cognitive impairment [5]. Elevated cytosolic $\mathrm{Ca}^{2+}$ levels induced by isoflurane has been demonstrated to activate the NF-kB signalling pathway [24] that stimulates the activation of pro-inflammatory cytokines [24]. We observed isoflurane exposure resulted in enhanced NF-kBp65, p-Iк-Ba, TNF- $\alpha$, IL-1 $\beta$ and IL- 6 expressions, in line with previous reports [5]. Paeonol at different tested doses markedly modulated NF-KB pathway by effectively down-regulating NF-kBp65, p-Ik-Ba, TNF- $\alpha, \quad I L-1 \quad \beta$ and IL-6 suggesting the involvement of NF-KB cascades in paeonol mediated neuroprotection.

The JNK signalling pathway has been documented to be involved in neurodegeneration in brain trigged by external stress such as ischemia/reperfusion and ethanol [25]. Previous studies have demonstrated activation of JNK pathway in isoflurane-induced apoptosis [11]. We observed strikingly enhanced phosphorylatedJNK on isoflurane exposure, suggesting activated JNK signals which is also supported by elevated expressions of $\mathrm{p}$-c-Jun, a JNK target protein. Furthermore, activated JNK modulates the expressions of $\mathrm{Bcl}-2$ proteins leading to apoptosis [25]. Bcl-2/Bcl-xL phosphorylation causes change in the mitochondrial membrane potential, leading to the release of cytochrome $C$. This subsequently activates caspase- 9 and caspase-3 leading to apoptosis. Thus paeonol mediated suppression of JNK activation critically inhibits release of cytochrome $\mathrm{C}$ and eventually apoptosis.

Furthermore it has been demonstrated that isoflurane can activate p38 MAPK [26]. Raised levels of p38 and p-p38 were observed in the present study, indicating activation of the pathway. Studies have shown that p38 MAPK signalling cascade exerts crucial roles in inflammatory process [26]. Thus the enhanced expressions could have also contributed to the inflammatory responses observed. Effective down-regulation of p-38 and p-p38 expression reveals inhibition of $p 38$ signalling on paeonol administration. However, although increases in the phosphorylated levels of ERK were noticed on isoflurane exposure, the elevations were not significant as seen in the levels of JNK and p38. Paeonol administration did not significantly cause any alterations on the activation levels of ERK. These observations suggest the involvement of JNK and p38MAPKs in isoflurane-induced toxicity with negligible involvement of ERK. 
Accumulating experimental data have raised concern over neuronal death and long-term cognitive dysfunctions and memory impairments induced by anesthetics in young rodent brains $[1,17]$. The observations in the open field test indicating improved behaviour on paeonol treatment. Working memory is associated in holding information temporarily in order to perform cognitive tasks that are considerably complex. Such tasks involve both hippocampus and prefrontal cortex [28]. Y-maze tests indicate profound alterations in the working memory on isoflurane exposure. Working memory is concerned with processes involving synchronized temporary storage for performing cognitive functions that demand intricate analysis and manipulation [28].. These data obtained through the behavioural analysis provide valuable insight into the effects of neonatal anesthesia exposure on cognitive capacities.

Furthermore, paeonol remarkably improved the behaviour and memory of the animals. The P35 rats exhibited a much better performance in $\mathrm{Y}$ maze tests. Paeonol-mediated reduction in hippocampal apoptosis and neuroinflammation could have possibly improved the performance.

At P7, rats are extremely sensitive to neurotoxic challenge [1] and thus exposure to isoflurane on P7 could have severely impaired neurogenesis. MWM is the most frequently employed test and also is a reliable measure of hippocampusdependent spatial navigation and memory assessment [22]. The rats were trained to explore the swimming pool and reach the submerged platform. As expected escape latencies of the rats that received isoflurane alone was significantly longer than the controls that were not exposed to anesthesia. More importantly, neuroinflammation also mediates isoflurane-induced cognitive impairment [5].

Nevertheless, we observed effective improvement in the working memory of the rats administered paeonol. At all the given doses, paeonol remarkably enhanced the performance of rats in MWM tests. The rats also exhibited improved memory retention. The results of the place, probe and cued trials suggest paeonol effectively enhanced the long-term memory of rats.

\section{CONCLUSION}

Significant inhibition of neurodegeneration, suppression of NF-KB signalling and marked modulation in the JNK/ERK/p38 MAPK pathways observed on paeonol treatment probably contribute to the improvement in learning and memory. Paeonol should therefore be further explored for other molecular events which may aid in the development of therapies against anesthetic-induced neuronal toxicities.

\section{DECLARATIONS}

\section{Conflict of Interest}

No conflict of interest associated with this work.

\section{Contribution of Authors}

The authors declare that this work was done by the authors named in this article and all liabilities pertaining to claims relating to the content of this article will be borne by them.

\section{REFERENCES}

1. Fang F, Xue Z, Cang J. Sevoflurane exposure in 7-dayold rats affects neurogenesis, neurodegeneration and neurocognitive function. Neurosci Bull 2012; 28: 499508.

2. Brambrink AM, Evers AS, Avidan MS, Farber NB, Smith DJ, Zhang $X$, Dissen GA, Creeley CE, Olney JW. Isoflurane-induced neuroapoptosis in the neonatal rhesus macaque brain. Anesthesiology 2010; 112: 834841.

3. Kong $F, X u L$, He D, Zhang $X, L u H$. Effects of gestational isoflurane exposure on postnatal memory and learning in rats. Eur J Pharmacol 2011; 670: 168-174.

4. DiMaggio C, Sun LS, Li G. Early childhood exposure to anesthesia and risk of developmental and behavioral disorders in a sibling birth cohort. Anesth Analg 2011; 113: 1143-1151.

5. Cao L, Li L, Lin D, Zuo Z. Isoflurane induces learning impairment that is mediated by interleukin $1 \beta$ in rodents. PLoS ONE 2012; 7: e51431.

6. Yon JH, Daniel-Johnson J, Carter LB, Jevtovic-Todorovic $\checkmark$. Anesthesia induces neuronal cell death in the developing rat brain via the intrinsic and extrinsic apoptotic pathways. Neuroscience 2005; 135: 815-827.

7. Mousa A, Bakhiet M. Role of cytokine signaling during nervous system development. Int J Mol Sci 2013; 14: 13931-13957.

8. Harper SJ, Wilkie N. MAPKs: new targets for neurodegeneration. Expert Opin Ther Targets 2003; 7: 187-200.

9. Kaminska B, Gozdz A, Zawadzka M, Ellert-Miklaszewska A, Lipko M. MAPK signal transduction underlying brain inflammation and gliosis as therapeutic target. Anat Rec (Hoboken) 2009; 292: 1902-1913.

10. Kuan CY, Burke RE. Targeting the JNK signaling pathway for stroke and Parkinson's diseases therapy. Curr Drug Targets CNS Neurol Disord 2005; 4: 63-67.

11. Cuadrado A, Nebreda AR. Mechanisms and functions of p38 MAPK signalling. Biochem J 2010; 429: 403-417.

Trop J Pharm Res, October 2016; 15(10): 2181 
12. Du Q, Feng GZ, Shen L, Cui J, Cai JK. Paeonol attenuates airway inflammation and hyperresponsiveness in a murine model of ovalbumin-induced asthma. Can J Physiol Pharmacol 2010; 88: 1010-1016.

13. Sun GP, Wan $X$, Xu SP, Wang H, Liu SH, Wang ZG. Anti-proliferation and apoptosis induction of paeonol in human esophageal cancer cell lines. Dis Esophagus 2008; 21: 723-729.

14. Wu JB, Song NN, Wei $X B$, Guan HS, Zhang $X M$. Protective effects of paeonol on cultured rat hippocampal neurons against oxygen-glucose deprivation-induced injury. J Neurol Sci 2008; 264: 5055.

15. Nizamutdinova IT, Jin YC, Kim JS, Yean MH, Kang SS, Kim YS, Lee JH, Seo HG, Kim HJ, Chang KC. Paeonol and Paeoniflorin, the main active principles of Paeonia albiflora, protect the heart from myocardial ischemia/reperfusion injury in rats. Planta Med 2008; 74: 14-18.

16. Garber JC. (Chair) Committee for the Update of the Guide for the Care and Use of Laboratory Animals. Guide for the Care and Use of Laboratory Animals, 8 edition. National Academy of Sciences. 2011.

17. Orliaguet G, Vivien B, Langeron $O$, Bouhemad B, Coriat $P$, Riou B. Minimum alveolar concentration of volatile anesthetics in rats during postnatal maturation. Anesthesiology 2001; 95: 34-39.

18. Li Y, Liang G, Wang S, Meng Q, Wang Q, Wei H. Effect of fetal exposure to isoflurane on postnatal memory and learning in rats. Neuropharmacology 2007; 53: 942-50.

19. Satoh $Y$, Endo S, Ikeda T, Yamada K, Ito M, Kuroki M, Hiramoto $T$, Imamura $O$, Kobayashi $Y$, Watanabe $Y$, et al. Extracellular signal-regulated kinase 2 (ERK2) knockdown mice show deficits in long-term memory; ERK2 has a specific function in learning and memory. J Neurosci 2007; 27: 10765-10776.

20. Kodama M, Satoh Y, Otsubo Y, Araki Y, Yonamine R, Masui K, Kazama T. Neonatal desflurane exposure induces more robust neuroapoptosis than do isoflurane and sevoflurane and impairs working memory. Anesthesiology 2011; 115: 979-991.

21. Culley DJ, Baxter MG, Yukhananov R, Crosby $G$. Impairment of acquisition of a spatial memory task following isoflurane-nitrous oxide anesthesia in rats. Anesthesiology 2004; 100: 309-314.

22. Istaphanous GK, Howard J, Nan X, Hughes EA, McCann JC, McAuliffe JJ, Danzer SC, Loepke AW. Comparison of the neuroapoptotic properties of equipotent anesthetic concentrations of desflurane, isoflurane, or sevoflurane in neonatal mice. Anesthesiology 2011; 114: 578-587.

23. Zhao $H$, Yenari MA, Cheng D, Sapolsky RM, Steinberg GK. Bcl-2 overexpression protects against neuron loss within the ischemic margin following experimental stroke and inhibits cytochrome $c$ translocation and caspase-3 activity. J Neurochem 2003; 85: 1026-1036.

24. Vexler ZS, Yenari MA. Does inflammation after stroke affect the developing brain differently than adult brain? Dev Neurosci 2009; 31: 378-393.

25. Guan QH, Pei DS, Zhang QG, Hao ZB, Xu TL, Zhang GY. The neuroprotective action of SP600125, a new inhibitor of JNK, on transient brain ischemia/reperfusioninduced neuronal death in rat hippocampal CA1 via nuclear and non-nuclear pathways. Brain Res 2005; 1035: 51-59.

26. Zheng S, Zuo Z. Isoflurane preconditioning induces neuroprotection against ischemia via activation of $P 38$ mitogen-activated protein kinases. Mol Pharmacol 2004; 65: 1172-1180.

27. Gentry KR, Steele LM, Sedensky MM, Morgan PG. Early developmental exposure to volatile anesthetics causes behavioral defects in Caenorhabditis elegans. Anesth Analg 2013; 116: 185-189.

28. Jones MW. A comparative review of rodent prefrontal cortex and working memory. Curr Mol Med 2002; 2: 639-647. 